\title{
Iron Project: atomic data for IR lines
}

\author{
N.R. Badnell ${ }^{1}$, M.A. Bautista ${ }^{2}$, K.A. Berrington ${ }^{3}$, V.M. Burke ${ }^{4}$, \\ K. Butler ${ }^{5}$, M.E. Galavís ${ }^{6}$, M. Graziani ${ }^{7}$, D.C. Griffin ${ }^{8}$, D.J. Lennon ${ }^{5} \dagger$, \\ C. Mendoza ${ }^{2}$, D.M. Mitnik ${ }^{8}$, J.C. Pelan ${ }^{9}$, A.K. Pradhan ${ }^{7}$, \\ H.E. Saraph ${ }^{10}$, P.J. Storey ${ }^{10}$, J.A. Tully ${ }^{11}$, C.J. Zeippen ${ }^{12}$, \\ and H.L. Zhang ${ }^{7}$ \\ ${ }^{1}$ Dept. of Physics, University of Strathclyde, Glasgow G4 0NG, UK \\ ${ }^{2}$ Centro de Física, IVIC, PO Box 21827, Caracas 1020A, Venezuela \\ ${ }^{3}$ School of Science \& Mathematics, Sheffield Hallam University, Sheffield S1 1WB, UK \\ ${ }^{4}$ CLRC Daresbury Laboratory, Warrington WA4 4AD, UK \\ ${ }^{5}$ Inst. für Astronomie und Astrophysik, Universität München, D-81679 München, Germany \\ ${ }^{6}$ Dept. de Física, Universidad Metropolitana, PO Box 76819, Caracas 1070A, Venezuela \\ ${ }^{7}$ Dept. of Astronomy, Ohio State University, Columbus, Ohio 43210, USA \\ ${ }^{8}$ Dept. of Physics, Rollins College, Winter Park, Florida 32789, USA \\ ${ }^{9} \mathrm{GCNU}$, University College London, London WC1N 3AR, UK \\ ${ }^{10}$ Dept. of Physics and Astronomy, University College London, London WC1E 6BT, UK \\ ${ }^{11}$ Dept. Cassiopée, Observatoire de la Côte d'Azur, BP 4229, 06304 Nice Cedex 4, France \\ ${ }^{12}$ LUTH, Observatoire de Paris, F-92195 Meudon, France
}

\begin{abstract}
The Iron Project is an international consortium dedicated to the computation of atomic data for astrophysical applications. Although the project has been mainly concerned with ions in the iron group, the earlier papers gave priority to calculations of $A$-values and electron impact collision strengths for infrared transitions. In the present report we include a compilation of these data which will become useful in the spectral modelling of planetary nebulae.
\end{abstract}

Keywords. Atomic data, infrared: general.

\section{Introduction}

The present report includes a compilation of radiative transition probabilities, $A$, and electron impact effective collision strengths, $\Upsilon(T)$, for the modelling of infrared transitions. They were calculated in the earlier stages of the Iron Project (IP, Hummer et al. 1993), a productive international collaboration dedicated to the computation of atomic data for astrophysical applications. To date comprising over 60 papers, the documentation, published in the A\&A series "Atomic data from the IRON Project", and the data sets can be downloaded from the TIPbase $\|$ online atomic database at the Centre de Données astronomiques de Strasbourg, France. In the IP considerable attention has been given to issues affecting data accuracy, using powerful numerical methods, well researched ionic target models and thorough data analyses. In Section 2 we give a brief description of the methods, followed by an explanation in Section 3 of the tables and their contents. These tables are also available in electronic form from TIPbase.

$\dagger$ Present address: Isaac Newton Group, Apdo. 321, 38700 Santa Cruz de La Palma, Spain.

$\ddagger$ Present address: Inst. de Astronomía y Física del Espacio and Dept. de Física, Universidad de Buenos Aires, CC67 Suc. 28, C1428EGA Buenos Aires, Argentina.

I Present address: MS F663, Los Alamos National Laboratory, Los Alamos, NM 87545, USA.

$$
\text { || http://vizier.u-strasbg.fr/tipbase/ }
$$




\section{Numerical Methods}

The $A$-values herein listed have been computed with an upgraded version of the atomic structure code SUPERSTRUCTURE (Eissner et al. 1974, Nussbaumer \& Storey 1974). The ionic wavefunctions take the form of configuration-interaction expansions of the type

$$
\Psi=\sum_{i} \phi_{i} c_{i}
$$

where the basis functions $\phi_{i}$ are constructed from single-electron orbitals generated in a Thomas-Fermi-Dirac model potential. Relativistic effects are taken into account with a Breit-Pauli hamiltonian; semi-empirical term-energy corrections are introduced before diagonalisation in order to improve wavefunction accuracy; and the transition probabilities are computed with the experimental level energy separations.

Electron collision rates for transitions between two ionic levels, $i$ and $j$ say $(j>i)$, can be expressed in $\mathrm{cm}^{3} \mathrm{~s}^{-1}$

$$
q(j, i ; T)=\frac{8.631 \times 10^{-6} \Upsilon(j, i ; T)}{\omega(j) T^{1 / 2}} \quad \text { and } \quad q(i, j)=\frac{\omega(j)}{\omega(i)} \exp \left[-\frac{\Delta E(i, j)}{\kappa T}\right] q(j, i)
$$

where $\omega(j)$ and $\omega(i)$ are the statistical weights of the upper and lower levels, respectively, $\Delta E(i, j)$ is the level energy separation, $T$ the electron temperature in $\mathrm{K}$ and $\kappa$ is the Boltzmann constant. The effective collision strength $\Upsilon(j, i ; T)$ is obtained by integrating the collision strength $\Omega(i, j ; E)$ over a Maxwellian distribution. Collision strengths for fine-structure levels have been computed in the close-coupling approximation with the $R$ matrix method (Berrington et al. 1978, Seaton 1985), followed by an algebraic recoupling of the $L S$ reactance matrices to intermediate coupling that can include target relativistic effects (Saraph 1978, Griffin et al. 1998); or alternatively, by the Breit-Pauli $R$-matrix method (Scott \& Burke 1980, Scott \& Taylor 1982). In these schemes, the wavefunction for an ionic target + electron system is expanded in terms of the target eigenfunctions

$$
\Psi=\mathcal{A} \sum_{i} \chi_{i} \theta_{i}+\sum_{j} c_{j} \Phi_{j}
$$

where $\mathcal{A}$ is the antisymmetrisation operator, $\chi_{i}$ are the target eigenfunctions, $\theta_{i}$ the electron functions, and $\Phi_{j}$ are bound-state type functions of the total system introduced to compensate for orthogonality conditions imposed on the $\theta_{i}$ and to render short-range correlations. In most cases, target level energies are corrected with the experimental values before hamiltonian diagonalisation.

\section{Table contents}

Table 1: For ions with nuclear charge $Z$ and electron number $N$, experimental energy levels, $E(Z, N, i)$, are tabulated from the following sources. $E(6-8, N, i)$ : Moore (1993). $E(10-16,5-8, i)$ : Edlén (1983a, 1983b, 1985). E(10,9, i): Kelly (1987). E(11-16,9-17, i): Martin \& Zalubas $(1979,1980,1981,1983)$ and Martin et al. (1990). E(18, 9-17, i): Shirai et al. (1999). E(20,9-17,i): Sugar \& Corliss (1985).

Table 2: $A$-values, $A(Z, N, j, i)$, and effective collision strengths, $\Upsilon(Z, N, j, i ; T)$, for the B, F, Al and $\mathrm{Cl}$ sequences. $A(6-16,5,2,1)$ : Galavís et al. (1998). $\Upsilon(6-7,5,2,1)$ : Blum \& Pradhan (1992). $(8,5,2,1)$ : Zhang et al. (1994). $\Upsilon(10,5,2,1)$ : Mitnik et al. (2001). $\Upsilon(12-16,5,2,1)$ : Zhang et al. (1994). $(10,9,2,1)$ : Griffin et al. (2001). $(11-16,9,2,1)$ : Saraph \& Tully (1994) and Berrington et al. (1998). $(14,13,2,1)$ : Dufton \& Kingston (1991) (these data are not associated with the IP but are included for completion). 
$\Upsilon(16-20,13,2,1)$ : Saraph \& Storey $(1996,1999) . \Upsilon(18-20,17,2,1)$ : Pelan \& Berrington (1995). $A(Z, N, 2,1)$ for $N=9, N=13$ and $N=17$ have been computed for this work.

Table 3: $A$-values and effective collision strengths for the $\mathrm{C}$ sequence. $A(7-16,6, j, i)$ : Galavís et al. (1997). $\Upsilon(7-16,6, j, i)$ : Lennon \& Burke (1994).

Table 4: $A$-values and effective collision strengths for the $\mathrm{O}$ sequence. $A(10-18,8, j, i)$ : Galavís et al. (1997). $\Upsilon(10-18,8, j, i)$ : Butler \& Zeippen (1994).

Table 5: $A$-values and effective collision strengths for the $\mathrm{Si}$ and $\mathrm{S}$ sequences. $A(16-$ 20,14, $j, i)$ : Mendoza \& Zeippen (1982). A(18-20,16, $j, i)$ : Mendoza \& Zeippen (1983). $\Upsilon(16-20,14, j, i)$ and $\Upsilon(18-20,16, j, i)$ : Galavís et al. (1995).

Note: Effective collision strengths for Fe II are given by Pradhan \& Zhang (1993) and Zhang \& Pradhan (1995) and for Ni II by Bautista (2004). Collisional data for neutral species have not been calculated in the IP. We nevertheless recommend the following sources. $\Upsilon(6,6, j, i)$ : Johnson et al. (1987) and Zatsarinny et al. (2005). $\Upsilon(8,8, j, i)$ : Bell et al. (1998) and Zatsarinny \& Tayal (2003). $(16,16, j, i)$ : Tayal (2004).

\section{References}

Bautista, M.A. 2004, A $\mathscr{E} A$ 420, 763

Bell, K.L., Berrington, K.A. \& Thomas, M.R.J. 1998, MNRAS 293, L83

Berrington, K.A., Burke, P.G., Le Dourneuf, M. et al. 1978, CoPhC 14, 367

Berrington, K.A., Saraph, H.E. \& Tully, J.A. 1998, A\&SAS 129, 161

Blum, R.D. \& Pradhan, A.K. 1992, ApJS 80, 425

Butler, K. \& Zeippen, C.J. 1994, A\&SAS 108, 1

Dufton, P.L. \& Kingston, A.E. 1991, MNRAS 248, 827

Edlén, B. 1983a, PhyS 28, 51

Edlén, B. 1983b, PhyS 28, 483

Edlén, B. 1985, PhyS 31, 345

Eissner, W., Jones, M. \& Nussbaumer, H. 1974, CoPhC 8, 270

Galavís, M.E., Mendoza, C. \& Zeippen, C.J. 1995, A\&AS 111, 347

Galavís, M.E., Mendoza, C. \& Zeippen, C.J. 1997, A\&AS 123, 159

Galavís, M.E., Mendoza, C. \& Zeippen, C.J. 1998, A\&AS 131, 499

Griffin, D.C., Badnell, N.R. \& Pindzola, M.S. 1998, JPhB 31, 3713

Griffin, D.C., Mitnik, D.M. \& Badnell, N.R. 2001, JPhB 34, 4401

Hummer, D.G., Berrington, K.A., Eissner, W. et al. 1993, A\&A 279, 298

Johnson, C.T., Burke, P.G. \& Kingston, A.E. 1987, JPhB 20, 2553

Kelly, R.L. 1987, JPCRD 16, Suppl. 1

Lennon, D.J. \& Burke, V.M. 1994, A\&AS 103, 273

Martin, W.C. \& Zalubas, R. 1979, JPCRD 8, 817

Martin, W.C. \& Zalubas, R. 1980, JPCRD 9, 1

Martin, W.C. \& Zalubas, R. 1981, JPCRD 10, 153

Martin, W.C. \& Zalubas, R. 1983, JPCRD 12, 323

Martin, W.C., Zalubas, R. \& Musgrove, A. 1990, JPCRD 19, 821

Mendoza, C. \& Zeippen, C.J. 1982, MNRAS 199, 1025

Mendoza, C. \& Zeippen, C.J. 1983, MNRAS 202, 981

Mitnik, D.M., Griffin, D.C. \& Badnell, N.R. 2001, JPhB 34, 4455

Moore, C.E. 1993, J.W. Gallagher (ed.), Tables of Spectra of Hydrogen, Carbon, Nitrogen, and Oxygen Atoms and Ions (Boca Raton: CRC Press)

Nussbaumer, H. \& Storey, P.J. 1978, AEGA 64, 139

Pelan, J. \& Berrington, K.A. 1995, A\& $A S$ 110, 209

Pradhan, A.K. \& Zhang, H.L. 1993, ApJ 409, L77

Saraph, H.E. 1978, CoPhC 15, 247

Saraph, H.E. \& Storey, P.J. 1996, A\&AS 115, 151

Saraph, H.E. \& Storey, P.J. 1999, A\&AS 134, 369 
Saraph, H.E. \& Tully, J.A. 1994, A\&SAS 107, 29

Scott, N.S. \& Burke, P.G. 1980, JPhB 13, 4299

Scott, N.S. \& Taylor, K.T. 1982, CoPhC 25, 347

Seaton, M.J. 1985, JPhB 18, 2111

Shirai, T., Sugar, J. \& Musgrove, A. 1999, unpublished

Sugar, J. \& Corliss, C. 1985, JPCRD 14, Suppl. 2

Tayal, S.S. 2004, ApJS 153, 581

Zatsarinny, O., Bartschat, K., Bandurina, L. \& Gedeon, V. 2005, PhRvA 71, 042702

Zatsarinny, O. \& Tayal, S.S. 2003, ApJS 148, 575

Zhang, H.L., Graziani, M. \& Pradhan, A.K. 1994, A\&A 283, 319

Zhang, H.L. \& Pradhan, A.K. 1995, A $\& A$ 293, 953

Table 1. Energy levels $\left(a \pm b \equiv a \times 10^{ \pm b}\right)$

\begin{tabular}{|c|c|c|c|c|c|c|c|c|}
\hline$(Z, N, i)$ & tate & d) & $\mid(Z, N, i)$ & te & d) & $(Z, N, i)$ & e & 1) \\
\hline & $\begin{array}{l}\mathrm{O} \\
1 / 2\end{array}$ & & 7 & $\mathrm{p}^{2}{ }^{1} \mathrm{~S}_{0}$ & 1 & & $2 p^{5}$ & \\
\hline & $\mathrm{p}^{2} \mathrm{P}_{3 / 2}^{\mathrm{o}}$ & $.77950-4$ & $4,6,1)$ & $2 \mathrm{p}^{2}{ }^{3} \mathrm{P}_{0}$ & & 2) & $2 \mathrm{p}^{5}{ }^{2} \mathrm{P}_{1 / 2}^{\mathrm{o}}$ & 2 \\
\hline 1) & $2 \mathrm{p}^{2} \mathrm{P}_{1 / 2}^{\mathrm{o}}$ & 0.00000 & $(14,6,2)$ & $2 \mathrm{p}^{2}{ }^{3} \mathrm{P}_{1}$ & $32013-2$ & $(13,9,1)$ & $2 \mathrm{p}^{5}{ }^{2} \mathrm{P}_{3 / 2}^{\mathrm{o}}$ & 00000 \\
\hline$, 5,2)$ & $2 \mathrm{p}^{2} \mathrm{P}_{3 / 2}^{\mathrm{o}}$ & $.58932-3$ & $(14,6,3)$ & $2 \mathrm{p}^{2}{ }^{3} \mathrm{P}_{2}$ & $84589-2$ & $(13,9,2)$ & $2 \mathrm{p}^{5}{ }^{2} \mathrm{P}_{1 / 2}^{\mathrm{o} / 2}$ & $.13665-2$ \\
\hline$(8,5,1)$ & $2 \mathrm{p}^{2} \mathrm{P}_{1 / 2}^{\mathrm{o}}$ & .00000 & $(14,6,4)$ & $2 \mathrm{p}^{2}{ }^{1} \mathrm{D}_{2}$ & $82316-1$ & $(14,9,1)$ & $2 \mathrm{p}^{5}{ }^{2} \mathrm{P}_{3 / 2}^{\mathrm{o}}$ & 00000 \\
\hline 2) & $2 \mathrm{p}^{2} \mathrm{P}_{3 / 2}^{\mathrm{o}}$ & $3.51670-3$ & $(14,6,5)$ & $2 \mathrm{p}^{2}{ }^{1} \mathrm{~S}_{0}$ & $82292-1$ & $(14,9,2)$ & $2 \mathrm{p}^{5}{ }^{2} \mathrm{P}_{1 / 2}^{\mathrm{o}}$ & $63844-2$ \\
\hline , 1) & $2 \mathrm{p}^{2} \mathrm{P}_{1 / 2}^{\mathrm{o} / 2}$ & 0.00000 & $(16,6,1)$ & $2 \mathrm{p}^{2}{ }^{3} \mathrm{P}_{0}$ & 00 & $(16,9,1)$ & $2 \mathrm{p}^{5}{ }^{2} \mathrm{P}_{3 / 2}^{\mathrm{o}}$ & 00000 \\
\hline $10,5,2)$ & $2 \mathrm{p}^{2} \mathrm{P}_{3 / 2}^{\mathrm{o}}$ & $1.19197-2$ & $(16,6,2)$ & $2 \mathrm{p}^{2}{ }^{3} \mathrm{P}_{1}$ & $5-2$ & $(16,9,2)$ & $2 \mathrm{p}^{5}{ }^{2} \mathrm{P}_{1 / 2}^{\mathrm{o}}$ & $9-2$ \\
\hline$(12,5,1)$ & $2 \mathrm{p}^{2} \mathrm{P}_{1 / 2}^{\mathrm{o}}$ & 000 & $(16,6,3)$ & $2 \mathrm{p}^{2}{ }^{3} \mathrm{P}_{2}$ & $90-1$ & $(14,13,1)$ & $3 \mathrm{p}^{2} \mathrm{P}_{1 / 2}^{\mathrm{o}}$ & 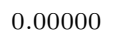 \\
\hline 2) & $2 \mathrm{p}^{2} \mathrm{P}_{3 / 2}^{\mathrm{o}}$ & $3.00907-2$ & $(16,6,4)$ & $2 \mathrm{p}^{2}{ }^{1} \mathrm{D}_{2}$ & $99-1$ & $(14,1$ & $3 \mathrm{p}^{2} \mathrm{P}_{3 / 2}^{\mathrm{o}}$ & $0-3$ \\
\hline 1) & $2 \mathrm{p}^{2} \mathrm{P}_{1 / 2}^{\mathrm{o}}$ & 0.00000 & $(16,6,5)$ & $2 \mathrm{p}^{2}{ }^{1} \mathrm{~S}_{0}$ & +0 & , 1) & $3 \mathrm{p}^{2} \mathrm{P}_{1 / 2}^{\mathrm{o}}$ & .00000 \\
\hline 2) & $2 \mathrm{p}^{2} \mathrm{P}_{3 / 2}^{\mathrm{o}}$ & $4.45619-2$ & $(10,8,1)$ & $2 \mathrm{p}^{4}{ }^{3} \mathrm{P}_{2}$ & & ,2) & $3 \mathrm{p}^{2} \mathrm{P}_{3 / 2}^{\mathrm{o}}$ & $22-3$ \\
\hline 1) & $2 \mathrm{p}^{2} \mathrm{P}_{1 / 2}^{\mathrm{o}}$ & 0.0 & ,2) & $2 \mathrm{p}^{4}{ }^{3} \mathrm{P}_{1}$ & -3 & , 1) & $3 \mathrm{p}^{2} \mathrm{P}_{1 / 2}^{\mathrm{o}}$ & 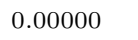 \\
\hline 2) & $2 \mathrm{p}^{2} \mathrm{P}_{3 / 2}^{\mathrm{o}}$ & $8-2$ & $(10$ & $2 \mathrm{p}^{4}{ }^{3} \mathrm{P}_{0}$ & -3 & & $3 \mathrm{p}^{2} \mathrm{P}_{3 / 2}^{\mathrm{o}}$ & $.01129-2$ \\
\hline 1) & $2 \mathrm{p}^{2} \mathrm{P}_{1 / 2}^{\mathrm{o}}$ & 0.00000 & (10 & $2 \mathrm{p}^{4}{ }^{1} \mathrm{D}_{2}$ & $7-1$ & 1) & $3 \mathrm{p}^{2} \mathrm{P}_{1 / 2}^{\mathrm{o}}$ & \\
\hline 2) & $2 \mathrm{p}^{2} \mathrm{P}_{3 / 2}^{\mathrm{o}}$ & $6-1$ & $(10$ & $2 \mathrm{p}^{4}{ }^{1} \mathrm{~S}_{0}$ & -1 & 2) & $3 \mathrm{p}^{2} \mathrm{P}_{3 / 2}^{\mathrm{o}}$ & $7-2$ \\
\hline & $2 \mathrm{p}^{2}{ }^{3} \mathrm{P}_{0}$ & 0.00000 & 1) & $2 \mathrm{p}^{4}{ }^{3} \mathrm{P}_{2}$ & & 1) & $3 \mathrm{p}^{2}{ }^{3} \mathrm{P}_{0}$ & \\
\hline 2) & $2 \mathrm{p}^{2}{ }^{3} \mathrm{P}_{1}$ & $4-4$ & 2) & $2 \mathrm{p}^{4}{ }^{3} \mathrm{P}_{1}$ & -2 & 2) & $3 \mathrm{p}^{2}{ }^{3} \mathrm{P}_{1}$ & $1-3$ \\
\hline & $2 \mathrm{p}^{2}{ }^{3} \mathrm{P}_{2}$ & $8-3$ & $(11,8,3)$ & $2 \mathrm{p}^{4}{ }^{3} \mathrm{P}_{0}$ & -2 & 3) & $3 \mathrm{p}^{2}{ }^{3} \mathrm{P}_{2}$ & -3 \\
\hline & $2 \mathrm{p}^{2}{ }^{1} \mathrm{D}_{2}$ & -1 & (11 & $2 \mathrm{p}^{4}{ }^{1} \mathrm{D}_{2}$ & -1 & 4) & $3 \mathrm{p}^{2}{ }^{1} \mathrm{D}_{2}$ & -1 \\
\hline & $2 \mathrm{p}^{2}{ }^{1} \mathrm{~S}_{0}$ & $5-1$ & $5)$ & $2 \mathrm{p}^{4}{ }^{1} \mathrm{~S}_{0}$ & -1 & 5) & $3 \mathrm{p}^{2}{ }^{1} \mathrm{~S}_{0}$ & -1 \\
\hline & $2 \mathrm{p}^{2}{ }^{3} \mathrm{P}_{0}$ & 0 & $(12$ & $2 \mathrm{p}^{4}{ }^{3} \mathrm{P}_{2}$ & & & $3 \mathrm{p}^{2}{ }^{3} \mathrm{P}_{0}$ & \\
\hline & $2 \mathrm{p}^{2}{ }^{3} \mathrm{P}_{1}$ & $39-3$ & $(12,8,2)$ & $2 \mathrm{p}^{4}{ }^{3} \mathrm{P}_{1}$ & -2 & 2) & $3 \mathrm{p}^{2}{ }^{3} \mathrm{P}_{1}$ & $8-3$ \\
\hline & $2 \mathrm{p}^{2}{ }^{3} \mathrm{P}_{2}$ & $016-3$ & $(12,8,3)$ & $2 \mathrm{p}^{4}{ }^{3} \mathrm{P}_{0}$ & -2 & ,3) & $3 \mathrm{p}^{2}{ }^{3} \mathrm{P}_{2}$ & -2 \\
\hline$(8,6,4)$ & $2 \mathrm{p}^{2}{ }^{1} \mathrm{D}_{2}$ & $0-1$ & $(12,8,4)$ & $2 \mathrm{p}^{4}{ }^{1} \mathrm{D}_{2}$ & -1 & , 4) & $3 \mathrm{p}^{2}{ }^{1} \mathrm{D}_{2}$ & -1 \\
\hline$(8,6,5)$ & $2 \mathrm{p}^{2}{ }^{1} \mathrm{~S}_{0}$ & $3.93551-1$ & $(12,8,5)$ & $2 \mathrm{p}^{4}{ }^{1} \mathrm{~S}_{0}$ & $7-1$ & $(18$ & $3 \mathrm{p}^{2}{ }^{1} \mathrm{~S}_{0}$ & $4-1$ \\
\hline$(10,6,1)$ & $2 \mathrm{p}^{2}{ }^{3} \mathrm{P}_{0}$ & 000 & $(13,8,1)$ & $2 p^{4}{ }^{3} \mathrm{P}_{2}$ & 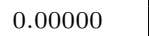 & $(20,14,1)$ & $3 \mathrm{p}^{2}{ }^{3} \mathrm{P}_{0}$ & \\
\hline, 2$)$ & $2 \mathrm{p}^{2}{ }^{3} \mathrm{P}_{1}$ & $3.76364-3$ & $(13,8,2)$ & $2 \mathrm{p}^{4}{ }^{3} \mathrm{P}_{1}$ & $4-2$ & $(20,14,2)$ & $3 \mathrm{p}^{2}{ }^{3} \mathrm{P}_{1}$ & $4-2$ \\
\hline & $2 \mathrm{p}^{2}{ }^{3} \mathrm{P}_{2}$ & $1.01245-2$ & $(13,8,3)$ & $2 \mathrm{p}^{4}{ }^{3} \mathrm{P}_{0}$ & -2 & $(20,14,3)$ & $3 \mathrm{p}^{2}{ }^{3} \mathrm{P}_{2}$ & $3-2$ \\
\hline & $2 \mathrm{p}^{2}{ }^{1} \mathrm{D}_{2}$ & $2.76039-1$ & $(13,8,4)$ & $2 \mathrm{p}^{4}{ }^{1} \mathrm{D}_{2}$ & $7-1$ & $(20,14,4)$ & $3 \mathrm{p}^{2}{ }^{1} \mathrm{D}_{2}$ & $2-1$ \\
\hline & $2 \mathrm{p}^{2}{ }^{1} \mathrm{~S}_{0}$ & $5.82452-1$ & $(13,8,5)$ & $2 \mathrm{p}^{4}{ }^{1} \mathrm{~S}_{0}$ & $09-1$ & $(20,14,5)$ & $3 \mathrm{p}^{2}{ }^{1} \mathrm{~S}_{0}$ & $7-1$ \\
\hline 3,1$)$ & $2 \mathrm{p}^{2}{ }^{3} \mathrm{P}_{0}$ & 0.00000 & $(14,8,1)$ & $2 p^{4}{ }^{3} \mathrm{P}_{2}$ & 0 & $(18,16,1)$ & $3 p^{4}{ }^{3} \mathrm{P}_{2}$ & \\
\hline 3,2$)$ & $2 \mathrm{p}^{2}{ }^{3} \mathrm{P}_{1}$ & $6.36080-3$ & $(14,8,2)$ & $2 \mathrm{p}^{4}{ }^{3} \mathrm{P}_{1}$ & $67066-2$ & $(18,16,2)$ & $3 p^{4}{ }^{3} \mathrm{P}_{1}$ & $51-2$ \\
\hline & $2 \mathrm{p}^{2}{ }^{3} \mathrm{P}_{2}$ & $1.69318-2$ & $(14,8,3)$ & $2 \mathrm{p}^{4}{ }^{3} \mathrm{P}_{0}$ & $3-2$ & $(18,16,3)$ & $3 p^{4}{ }^{3} \mathrm{P}_{0}$ & $2-2$ \\
\hline & $2 \mathrm{p}^{2}{ }^{1} \mathrm{D}_{2}$ & $3.23562-1$ & $(14,8,4)$ & $2 \mathrm{p}^{4}{ }^{1} \mathrm{D}_{2}$ & -1 & 4) & $3 p^{4}{ }^{1} D_{2}$ & $0-1$ \\
\hline & $2 \mathrm{p}^{2}{ }^{1} \mathrm{~S}_{0}$ & $8-1$ & & $2 \mathrm{p}^{4}{ }^{1} \mathrm{~S}_{0}$ & $8-1$ & & $3 p^{4}{ }^{1} S_{0}$ & $44-1$ \\
\hline & $2 \mathrm{p}^{2}{ }^{3} \mathrm{P}_{0}$ & & & $2 p^{4}{ }^{3} \mathrm{P}_{2}$ & & & $3 p^{4}{ }^{3} \mathrm{P}_{2}$ & \\
\hline & $2 \mathrm{p}^{2}{ }^{3} \mathrm{P}_{1}$ & & & $2 \mathrm{p}^{4}{ }^{3} \mathrm{P}_{1}$ & $59-2$ & & $3 p^{4}{ }^{3} \mathrm{P}_{1}$ & $35-2$ \\
\hline & $2 \mathrm{p}^{2}{ }^{3} \mathrm{P}_{2}$ & $1-2$ & & $2 \mathrm{p}^{4}{ }^{3} \mathrm{P}_{0}$ & $34-2$ & & $3 \mathrm{p}^{4}{ }^{3} \mathrm{P}_{0}$ & $9-2$ \\
\hline & $2 \mathrm{p}^{2}{ }^{1} \mathrm{D}_{2}$ & $3.73236-1$ & 4) & $2 \mathrm{p}^{4}{ }^{1} \mathrm{D}_{2}$ & $32-1$ & 4) & $3 p^{4}{ }^{1} D_{2}$ & $71597-1$ \\
\hline & $2 \mathrm{p}^{2}{ }^{1} \mathrm{~S}_{0}$ & $7.76080-1$ & 5) & $2 \mathrm{p}^{4}{ }^{1} \mathrm{~S}_{0}$ & $14+0$ & & $3 \mathrm{p}^{4}{ }^{1} \mathrm{~S}_{0}$ & $9473-1$ \\
\hline & $2 \mathrm{p}^{2}{ }^{3} \mathrm{P}_{0}$ & 0.00000 & $(10,9,1)$ & $2 \mathrm{p}^{5}{ }^{2} \mathrm{P}_{3 / 2}^{\mathrm{o}}$ & 0.00000 & 1) & $3 \mathrm{p}^{5}{ }^{2} \mathrm{P}_{3 / 2}^{\mathrm{o}}$ & 0.00000 \\
\hline 2) & $2 \mathrm{p}^{2}{ }^{3} \mathrm{P}_{1}$ & $1.56286-2$ & $(10,9,2)$ & $2 \mathrm{p}^{5}{ }^{2} \mathrm{P}_{1 / 2}^{\mathrm{o}}$ & $7.11117-3$ & $(18,17,2)$ & $3 \mathrm{p}^{5}{ }^{2} \mathrm{P}_{1 / 2}^{\mathrm{o}}$ & $1.30457-2$ \\
\hline$(13,6,3)$ & $2 \mathrm{p}^{2}{ }^{3} \mathrm{P}_{2}$ & $4.02697-2$ & $(11,9,1)$ & $2 \mathrm{p}^{5}{ }^{2} \mathrm{P}$ & 0.00000 & $(20,17,1)$ & $3 \mathrm{p}^{5}{ }^{2} \mathrm{P}_{3}^{\mathrm{o}}$ & 0.00000 \\
\hline$(13,6,4)$ & $2 \mathrm{p}^{2}{ }^{1} \mathrm{D}_{2}$ & $4.25835-1$ & $(11,9,2)$ & $2 \mathrm{p}^{5}{ }^{2} \mathrm{P}_{1 / 2}^{\mathrm{o}}$ & $1.24509-2$ & $(20,17,2)$ & $3 \mathrm{p}^{5}{ }^{2} \mathrm{P}_{1 / 2}^{\mathrm{o}}$ & $2.84155-2$ \\
\hline
\end{tabular}


Table 2. $A$-values and $\Upsilon(\log T)$ for the $\mathrm{B}, \mathrm{F}, \mathrm{Al}$ and $\mathrm{Cl}$ sequences $\left(a \pm b \equiv a \times 10^{ \pm b}\right)$

\begin{tabular}{|c|c|c|c|c|c|c|c|c|c|c|c|}
\hline \multirow[t]{2}{*}{$(Z, N, j, i)$} & \multirow[t]{2}{*}{$A\left(\mathrm{~s}^{-1}\right)$} & \multicolumn{10}{|c|}{$\Upsilon(\log T)$} \\
\hline & & 3.00 & 3.30 & 3.48 & 3.60 & 3.70 & 3.90 & 4.00 & 4.20 & 4.41 & 4.60 \\
\hline \multirow[t]{2}{*}{$(6,5,2,1)$} & $2.29-6$ & $1.58+0$ & $1.64+0$ & $1.72+0$ & $1.80+0$ & $1.89+0$ & $2.08+0$ & $2.15+0$ & $2.26+0$ & $2.28+0$ & $2.25+0$ \\
\hline & & 3.00 & 3.30 & 3.48 & 3.60 & 3.70 & 3.90 & 4.00 & 4.20 & 4.41 & 4.60 \\
\hline \multirow[t]{2}{*}{$(7,5,2,1)$} & $4.74-5$ & $1.29+0$ & $1.29+0$ & $1.29+0$ & $1.30+0$ & $1.32+0$ & $1.39+0$ & $1.45+0$ & $1.57+0$ & $1.73+0$ & $1.91+0$ \\
\hline & & 2.95 & 3.43 & 3.65 & 3.86 & 4.13 & 4.35 & 4.65 & 4.91 & 5.16 & 5.61 \\
\hline \multirow[t]{2}{*}{$(8,5,2,1)$} & $5.17-4$ & $1.61+0$ & $1.80+0$ & $1.96+0$ & $2.24+0$ & $2.51+0$ & $2.59+0$ & $2.69+0$ & $2.64+0$ & $2.32+0$ & $1.46+0$ \\
\hline & & 3.40 & 3.70 & 4.00 & 4.40 & 4.70 & 5.00 & 5.40 & 5.70 & 6.00 & 6.40 \\
\hline \multirow[t]{2}{*}{$(10,5,2,1)$} & $2.01-2$ & $3.61+0$ & $3.19+0$ & $2.61+0$ & $1.92+0$ & $1.75+0$ & $1.70+0$ & $1.38+0$ & $1.03+0$ & $7.22-1$ & $4.32-1$ \\
\hline & & 3.69 & 4.17 & 4.39 & 4.59 & 4.87 & 5.09 & 5.39 & 5.64 & 5.89 & 6.34 \\
\hline \multirow{2}{*}{$(12,5,2,1)$} & $3.24-1$ & $8.52-1$ & $1.12+0$ & $1.06+0$ & $1.01+0$ & $1.02+0$ & $1.05+0$ & $3-1$ & $7.81-1$ & $5.83-1$ & $3.18-1$ \\
\hline & & 3.8 & 4.2 & 4.51 & 4.7 & 4.98 & 5.20 & 5.51 & 5.76 & 6.01 & 6. \\
\hline \multirow[t]{2}{*}{$(13,5,2,1)$} & $1.05+0$ & $5.11-1$ & $4.82-1$ & $7.01-1$ & $9.50-1$ & $1.16+0$ & $1.18+0$ & $1.02+0$ & $7.85-1$ & $5.62-1$ & $2.90-1$ \\
\hline & & 3.91 & 4.39 & 4.61 & 4.81 & 5.09 & 5.31 & 5.61 & 5.86 & 6.11 & 6.56 \\
\hline \multirow[t]{2}{*}{$(14,5,2,1)$} & $3.07+0$ & $1.14+0$ & $1.59+0$ & $1.57+0$ & $1.44+0$ & $1.23+0$ & $1.07+0$ & $8.17-1$ & $5.99-1$ & $4.20-1$ & $2.16-1$ \\
\hline & & 4.08 & 4.56 & 4.78 & 4.99 & 5.26 & 5.48 & 5.63 & 6.04 & 6.29 & 6.74 \\
\hline \multirow[t]{2}{*}{$(16,5,2,1)$} & $2.04+1$ & $1.20-1$ & $2.98-1$ & $4.75-1$ & $6.09-1$ & $7.00-1$ & $6.89-1$ & $6.40-1$ & $4.18-1$ & $2.94-1$ & $1.53-1$ \\
\hline & & 3.0 & 3.3 & 3.6 & & 4.00 & 4.3 & 4.6 & 4.9 & 5.0 & \\
\hline \multirow[t]{2}{*}{$(10,9,2,1)$} & 8.5 & $2.66-1$ & $2.86-1$ & $2.99-1$ & $3.10-1$ & $3.14-1$ & $3.29-1$ & $3.50-1$ & $3.85-1$ & $4.00-1$ & $4.45-1$ \\
\hline & & 3.00 & 3.25 & 3.50 & 3.75 & 4.00 & 4.25 & 4.50 & 4.75 & 5.00 & \\
\hline \multirow[t]{2}{*}{$(11,9,2,1)$} & $4.58-2$ & $3.50-1$ & $3.50-1$ & $3.50-1$ & $3.52-1$ & $3.54-1$ & $3.57-1$ & $3.63-1$ & $3.76-1$ & $4.00-1$ & \\
\hline & & 3.00 & 3.25 & 3.5 & 3.75 & 4.00 & 4.20 & & 4.60 & 4.80 & 5.0 \\
\hline \multirow{2}{*}{$(12,9,2,1)$} & $.99-1$ & $3.56-1$ & $3.56-1$ & -1 & $3.56-1$ & $3.57-1$ & $3.58-1$ & $3.61-1$ & $3.68-1$ & $3.83-1$ & $4.05-$ \\
\hline & & 3.0 & 3.2 & 3. & 3. & 4.00 & 4.25 & 4.4 & 4.60 & 4.80 & 5. \\
\hline \multirow[t]{2}{*}{$(13,9,2,1)$} & $7.33-1$ & $3.81-1$ & $3.87-1$ & $4.03-1$ & $4.50-1$ & $5.07-1$ & $5.24-1$ & $5.14-1$ & $4.90-1$ & $4.70-1$ & $4.64-1$ \\
\hline & & $3.00+0$ & $3.25+0$ & $3.50+0$ & $3.75+0$ & $4.00+0$ & $4.25+0$ & $4.50+0$ & $4.56+0$ & $4.76+0$ & $4.96+0$ \\
\hline \multirow[t]{2}{*}{$(14,9,2,1)$} & $2.37+0$ & $3.01-1$ & $3.01-1$ & $2.99-1$ & $2.98-1$ & $2.96-1$ & $2.98-1$ & $3.22-1$ & $3.33-1$ & $3.79-1$ & $4.18-1$ \\
\hline & & 3.5 & 3.7 & 4.0 & 4.25 & 4.50 & 4.75 & 4.81 & 5.01 & 5.21 & 5.41 \\
\hline \multirow[t]{2}{*}{$(16,9,2,1)$} & $1.84+1$ & $1.92-1$ & $1.92-1$ & $1.92-1$ & $1.96-1$ & $2.09-1$ & $2.34-1$ & $2.41-1$ & $2.68-1$ & $2.88-1$ & $2.97-1$ \\
\hline & & 3.60 & 3.80 & 4.00 & 4.20 & 4.40 & 4.60 & & & & \\
\hline \multirow[t]{2}{*}{$(14,13,2,1)$} & $2.13-4$ & $5.58+0$ & $5.61+0$ & $5.70+0$ & $5.79+0$ & $5.75+0$ & $5.47+0$ & & & & \\
\hline & & 3.00 & 3.30 & 3.48 & 3.60 & 3.78 & 4.00 & 4.1 & 4. & 4. & 4. \\
\hline \multirow[t]{2}{*}{$(16,13,2,1)$} & $3-3$ & $6.89+0$ & $7.11+0$ & $7.52+0$ & $7.85+0$ & $8.27+0$ & $8.55+0$ & $8.51+0$ & $8.44+0$ & $7.92+0$ & $7.47+0$ \\
\hline & & 3.00 & 3.30 & 3.48 & 3.60 & 3.78 & 4.00 & 4.15 & 4.20 & 4.45 & 4.60 \\
\hline \multirow[t]{2}{*}{$(18,13,2,1)$} & $9.66-2$ & $3.11+0$ & $3.40+0$ & $3.86+0$ & $4.35+0$ & $5.13+0$ & $5.90+0$ & $6.19+0$ & $6.26+0$ & $6.36+0$ & $6.33+0$ \\
\hline & & $3.00+0$ & $3.30+0$ & $3.48+0$ & $3.60+0$ & $3.78+0$ & $4.00+0$ & $4.15+0$ & $4.20+0$ & $4.45+0$ & $4.60+0$ \\
\hline \multirow[t]{2}{*}{$(20,13,2,1)$} & $7.18-1$ & $2.66+0$ & $3.49+0$ & $4.28+0$ & $4.99+0$ & $5.98+0$ & $6.72+0$ & $6.72+0$ & $6.64+0$ & $6.10+0$ & $5.88+0$ \\
\hline & & 3.20 & 3.40 & 3.60 & 3.80 & 4.00 & 4.20 & 4.40 & 4.60 & 4.80 & 5.00 \\
\hline \multirow[t]{2}{*}{$(18,17,2,1)$} & $5.27-2$ & $2.48+0$ & $2.54+0$ & $2.63+0$ & $2.77+0$ & $2.93+0$ & $3.09+0$ & $3.19+0$ & $3.20+0$ & $3.13+0$ & $2.97+0$ \\
\hline & & 4.0 & 4.2 & 4.4 & 4.6 & & 5.00 & 5.20 & 5.40 & 5.60 & 6.00 \\
\hline$(20,17,2,1)$ & $5.45-1$ & $1.00+0$ & $1.12+0$ & $1.35+0$ & $1.77+0$ & $2.39+0$ & $2.80+0$ & $2.98+0$ & $2.82+0$ & $2.43+0$ & $1.53+0$ \\
\hline
\end{tabular}

Table 3. $A$-values and $\Upsilon(\log T)$ for the $\mathrm{C}$ sequence $\left(a \pm b \equiv a \times 10^{ \pm b}\right)$

\begin{tabular}{llllllllllll}
\hline$(Z, N, j, i)$ & $A\left(\mathrm{~s}^{-1}\right)$ & \multicolumn{10}{c}{$\Upsilon(\log T)$} \\
\cline { 3 - 12 } & & 3.00 & 3.20 & 3.40 & 3.60 & 3.80 & 4.00 & 4.20 & 4.40 & 4.60 & 5.00 \\
\hline$(7,6,2,1)$ & $2.08-6$ & $3.42-1$ & $3.37-1$ & $3.41-1$ & $3.59-1$ & $3.84-1$ & $4.08-1$ & $4.29-1$ & $4.57-1$ & $4.91-1$ & $5.43-1$ \\
$(7,6,3,1)$ & $0.00+0$ & $2.19-1$ & $2.21-1$ & $2.27-1$ & $2.35-1$ & $2.50-1$ & $2.72-1$ & $3.01-1$ & $3.31-1$ & $3.53-1$ & $3.49-1$ \\
$(7,6,3,2)$ & $7.46-6$ & $9.19-1$ & $9.18-1$ & $9.34-1$ & $9.77-1$ & $1.04+0$ & $1.12+0$ & $1.21+0$ & $1.32+0$ & $1.41+0$ & $1.46+0$ \\
$(7,6,4,1)$ & $3.55-7$ & $2.76-1$ & $2.77-1$ & $2.79-1$ & $2.83-1$ & $2.88-1$ & $2.93-1$ & $3.00-1$ & $3.07-1$ & $3.16-1$ & $3.29-1$ \\
$(7,6,4,2)$ & $1.02-3$ & $8.27-1$ & $8.31-1$ & $8.38-1$ & $8.49-1$ & $8.63-1$ & $8.80-1$ & $9.00-1$ & $9.22-1$ & $9.49-1$ & $9.87-1$ \\
$(7,6,4,3)$ & $3.01-3$ & $1.38+0$ & $1.38+0$ & $1.40+0$ & $1.41+0$ & $1.44+0$ & $1.47+0$ & $1.50+0$ & $1.54+0$ & $1.58+0$ & $1.64+0$ \\
$(7,6,5,1)$ & $0.00+0$ & $3.12-2$ & $3.13-2$ & $3.15-2$ & $3.17-2$ & $3.20-2$ & $3.26-2$ & $3.33-2$ & $3.44-2$ & $3.60-2$ & $3.91-2$ \\
$(7,6,5,2)$ & $3.30-2$ & $9.37-2$ & $9.40-2$ & $9.44-2$ & $9.51-2$ & $9.61-2$ & $9.77-2$ & $9.99-2$ & $1.03-1$ & $1.08-1$ & $1.17-1$ \\
$(7,6,5,3)$ & $1.31-4$ & $1.56-1$ & $1.57-1$ & $1.57-1$ & $1.59-1$ & $1.60-1$ & $1.63-1$ & $1.67-1$ & $1.72-1$ & $1.80-1$ & $1.96-1$ \\
$(7,6,5,4)$ & $1.02+0$ & $1.16+0$ & $1.13+0$ & $1.07+0$ & $1.00+0$ & $9.18-1$ & $8.34-1$ & $7.61-1$ & $7.10-1$ & $6.82-1$ & $6.59-1$ \\
\hline
\end{tabular}


Table 3. Continued

\begin{tabular}{|c|c|c|c|c|c|c|c|c|c|c|c|}
\hline \multirow[t]{2}{*}{$(Z, N, j, i)$} & \multirow[t]{2}{*}{$A\left(\mathrm{~s}^{-1}\right)$} & \multicolumn{10}{|c|}{$\Upsilon(\log T)$} \\
\hline & & 00 & 0 & 0 & 0 & 80 & 4.00 & 20 & 0 & 50 & 0 \\
\hline$(8$ & & & -1 & -1 & -1 & -1 & -1 & -1 & -1 & $9-1$ & $3-1$ \\
\hline & & & & & & & $2.71-1$ & & & & \\
\hline & & & & & & & & & & & \\
\hline & & & & & & & & & & & \\
\hline & & & & & & & & & & & \\
\hline & 04 & & & & & $1.20+0$ & $1.27+0$ & & & & $8+0$ \\
\hline & & & & & & $3.05-2$ & $3.25-2$ & & & & \\
\hline & $21-1$ & & & & & & $9.75-2$ & & & & \\
\hline & & & & & & & & & & & \\
\hline & & & & & & & & & & & \\
\hline & & & & & & & & & & & \\
\hline & & & & & & & & & & & \\
\hline & & & & & & & & & & & \\
\hline & & & & & & -1 & 1 & & & & \\
\hline & & & & & & & & & & & \\
\hline & 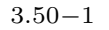 & & & & & & & & & & \\
\hline & & & & & & & & & & & \\
\hline & & & & & & & 8.1 & & & & \\
\hline & & & & & & & & & & & \\
\hline & & & & & & & & & & & \\
\hline & & & & & & & & & & & \\
\hline (11, & $70 \quad-8$ & & & & & & & & & & \\
\hline & & & & & & & & & & & \\
\hline & & & & & & & & & & & \\
\hline & & & & & & & & & & & \\
\hline & & & & & & & & & & & \\
\hline$(11,6$ & - & & & & & & & & & & \\
\hline & & & & & & & & & & & \\
\hline & & & & & & & & & & & \\
\hline & & & & & & & & & & & \\
\hline & & & & & & & & & & & \\
\hline 3 & 22 & & & & & & & & & & \\
\hline$(12$, & $05-2$ & & & & & & 108 & & & & +0 \\
\hline & & & & & & & & & & & \\
\hline & & & & & & & & & & & \\
\hline & & & & & & & & & & & \\
\hline & & & & & & & & & & & \\
\hline & & & & & & & & & & & \\
\hline & & & & & & & & & & & \\
\hline & & & & & & & & & & & \\
\hline & & & & & & & & & & & \\
\hline & & & & & & & & & & & \\
\hline & & & & & & & & & & & +0 \\
\hline 4,1$)$ & & & & & & & & & & & \\
\hline & & & & & & & & & & & \\
\hline & & & & & & & & & & & \\
\hline & & & & & & & & & & & \\
\hline & & & & & & & & & & & \\
\hline 5,3$)$ & & & & & & & & & & & -2 \\
\hline & & & & & & & & & & & \\
\hline & & & & & & & & & & & \\
\hline & & & & & & & & & & & \\
\hline & & & & & & & & & & & \\
\hline & & & & & & & & & & & -2 \\
\hline & & & & & & -1 & & & & -1 & $3-1$ \\
\hline & & & & & & & & & & & $2-1$ \\
\hline & & & & & & & & & & & \\
\hline & & & & & & & & & & & \\
\hline & & & & & & & & & & & -2 \\
\hline ,4) & & & & & & -2 & -2 & & & -1 & $5-1$ \\
\hline & & & & & & -1 & & & & -1 & $.18-1$ \\
\hline & & & & & & & & & & & \\
\hline & & & & & & & & & & & \\
\hline & & & & & & & & & & & \\
\hline & 70 & & $6-1$ & -1 & & $3-1$ & $1.91-1$ & -1 & 2.0 & -1 & $.81-1$ \\
\hline 3) & & & -1 & & & $2.88-1$ & & -1 & & $6-1$ & $01-1$ \\
\hline & & & & & & $5-3$ & 7.2 & & & $7-3$ & ent \\
\hline & & & & & & & & & & & \\
\hline & & & & & & & & & & & \\
\hline$(16,6,5,4)$ & $.48+0$ & $1.08-1$ & $1.17-1$ & $1.58-1$ & $2.72-1$ & $4.28-1$ & $5.41-1$ & $5.59-1$ & $5.01-1$ & $4.09-1$ & $2.62-1$ \\
\hline
\end{tabular}


Table 4. $A$-values and $\Upsilon(\log T)$ for the $\mathrm{O}$ sequence $\left(a \pm b \equiv a \times 10^{ \pm b}\right)$

\begin{tabular}{|c|c|c|c|c|c|c|c|c|c|c|c|}
\hline \multirow[t]{2}{*}{$(Z, N, j, i)$} & \multirow[t]{2}{*}{$A\left(\mathrm{~s}^{-1}\right)$} & \multicolumn{10}{|c|}{$\Upsilon(\log T)$} \\
\hline & & 3.00 & 3.20 & 3.40 & 60 & 3.80 & 4.00 & 20 & 10 & 60 & 00 \\
\hline & & -1 & & & -1 & $2-1$ & -1 & -1 & $1-1$ & $4-1$ & $.08-1$ \\
\hline & & & & & & & & & & & \\
\hline & 3 & & & & & & & & & & \\
\hline & & & & & & & & & & & \\
\hline & & & & & & & $2-1$ & & & & \\
\hline & & & & & & $.52-1$ & $.51-1$ & & & & \\
\hline & & & & & & & & & & & \\
\hline & & & & & & & & & & & \\
\hline & $0+0$ & & & & & & & & & & \\
\hline & & & & & & & & & & & \\
\hline & & & & & & & & & & & \\
\hline & & & & & & -1 & & & & & \\
\hline & & & & & & $9-1$ & & & & & \\
\hline & & & & & & & & & & & \\
\hline & & & & & & & & & & & \\
\hline$(11,8,4,3)$ & O & & & & & & & & & & \\
\hline & & & & & & & & & & & \\
\hline & & & & & & & & & & & \\
\hline & & & & & & & & & & & \\
\hline & & & & & & & & & & & \\
\hline & & & & & & & & & & & \\
\hline$(12$, & & & & & & & & & & & \\
\hline & & & & & & & & & & & \\
\hline & & & & & & & & & & & \\
\hline & & & & & & & & & & & \\
\hline & & & & & & & & & & & \\
\hline$(12,8,5,1)$ & $50-2$ & & & & & & & & & & \\
\hline & & & & & & & & & & & \\
\hline & & & & & & & & & & & \\
\hline & & & & & & & & & & & \\
\hline & & & & & & & & & & & \\
\hline & & & & & & & & & & & \\
\hline 3,2$)$ & $05-2$ & & & & & & & & & & \\
\hline & & & & & & & & & & & \\
\hline & & & & & & & & & & & \\
\hline & & & & & & & & & & & \\
\hline & & & & & & & & & & & \\
\hline & & & & & & & & & & & \\
\hline & & & & & & & & & & & \\
\hline & & & & & & & & & & & \\
\hline & & & & & & & & & & & \\
\hline & & & & & & & & & & & \\
\hline & & & & & & & & & & & \\
\hline & & & & & & & & & & & \\
\hline & & & & & & & & & & & \\
\hline & & & & & & & & & & & \\
\hline & & & & & & & & & & & \\
\hline$(1$ & & & & & & & & & & & \\
\hline$(14,8,5,3)$ & $00+0$ & & & & & & & & & -2 & $5-2$ \\
\hline & & & & & & & & & & & \\
\hline & & & & & & & & & & & \\
\hline & & & & & & & & & & & \\
\hline & & & & & & & & & & & \\
\hline$, 8,4,1)$ & & & & & & & & & & & -1 \\
\hline 4,2$)$ & & & & & & -1 & -1 & -1 & & -1 & $9-1$ \\
\hline & & & & & & & & & & & $5-2$ \\
\hline & & & & & & & & & & & \\
\hline & & & & & & & & & & & \\
\hline & .00 & & & & & & & & & & \\
\hline & & & & & & & & & & -1 & -1 \\
\hline 2,1$)$ & $68+1$ & -1 & $2.19-1$ & $2-1$ & & $1-1$ & & -1 & & -1 & $9-1$ \\
\hline $8,3,1)$ & & & & & & & & & & & $70-1$ \\
\hline & & & & & & & & & & & \\
\hline & & & & & & & & & & & \\
\hline & 00 & & & & & & & & & & -1 \\
\hline & 71 & & & & & & & & & & $12-2$ \\
\hline 5,1$)$ & $.20-1$ & 2 & -2 & -2 & -2 & $9-2$ & -2 & -2 & $1-2$ & -2 & $2.76-2$ \\
\hline & & & & & & & & & & & \\
\hline & & & & & & & & & & & \\
\hline$(18,8,5,4)$ & $9.69+0$ & $4.91-2$ & $4.92-2$ & $4.92-2$ & $4.93-2$ & $4.95-2$ & $5.00-2$ & $5.16-2$ & $5.59-2$ & $6.48-2$ & $9.78-$ \\
\hline
\end{tabular}


Table 5. $A$-values and $\Upsilon(\log T)$ for the Si and S sequences $\left(a \pm b \equiv a \times 10^{ \pm b}\right)$

\begin{tabular}{|c|c|c|c|c|c|c|c|c|c|c|c|}
\hline \multirow[t]{2}{*}{$(Z, N, j, i)$} & \multirow[t]{2}{*}{$A\left(\mathrm{~s}^{-1}\right)$} & \multicolumn{10}{|c|}{$\Upsilon(\log T)$} \\
\hline & & 3.00 & 3.20 & 3.40 & 3.60 & 3.80 & 00 & 4.20 & 4.40 & 4.60 & 5.00 \\
\hline 1) & $2-4$ & $2+0$ & $7+0$ & +0 & $6+0$ & 0 & $3+0$ & $28+0$ & $18+0$ & $8+0$ & -0 \\
\hline & $4.61-8$ & $8.03-1$ & $8.10-1$ & $8.66-1$ & $9.58-1$ & $1.04+0$ & $1.11+0$ & $1.21+0$ & $1.33+0$ & $1.41+0$ & $1.31+0$ \\
\hline$(16,14,3,2)$ & $2.07-3$ & $3.96+0$ & $4.16+0$ & $4.45+0$ & $4.85+0$ & $5.20+0$ & $5.41+0$ & $5.56+0$ & $5.71+0$ & $5.78+0$ & $5.10+0$ \\
\hline$(16,14,4,1)$ & $5.82-6$ & $6.29-1$ & $7.44-1$ & $8.37-1$ & $8.82-1$ & $8.85-1$ & $8.79-1$ & $8.85-1$ & $8.88-1$ & $8.69-1$ & $7.19-1$ \\
\hline$(16,14,4,2)$ & $2.21-2$ & $1.89+0$ & $2.23+0$ & $2.51+0$ & $2.65+0$ & $2.65+0$ & $2.64+0$ & $2.65+0$ & $2.66+0$ & $2.61+0$ & $2.16+0$ \\
\hline $4,4,3)$ & $5.76-2$ & $3.14+0$ & $3.72+0$ & $4.19+0$ & $4.41+0$ & $4.42+0$ & $4.39+0$ & $4.42+0$ & $4.44+0$ & $4.34+0$ & $3.60+0$ \\
\hline $4,5,1)$ & $0.00+0$ & $1.39-1$ & $1.35-1$ & $1.29-1$ & $1.24-1$ & $1.22-1$ & $1.22-1$ & $1.25-1$ & $1.32-1$ & $1.40-1$ & $1.27-1$ \\
\hline$(16,14,5,2)$ & $7.96-1$ & $4.17-1$ & $4.06-1$ & $3.88-1$ & $3.73-1$ & $3.66-1$ & $3.66-1$ & $3.75-1$ & $3.96-1$ & $4.19-1$ & $3.82-1$ \\
\hline$(16,14,5,3)$ & $1.05-2$ & $6.95-1$ & $6.77-1$ & $6.47-1$ & $6.21-1$ & $6.09-1$ & $6.10-1$ & $6.24-1$ & $6.60-1$ & $6.99-1$ & $6.36-1$ \\
\hline$(16,14,5,4)$ & $2.22+0$ & $9.93-1$ & $9.72-1$ & $9.43-1$ & $9.56-1$ & $1.08+0$ & $1.29+0$ & $1.53+0$ & $1.77+0$ & $1.96+0$ & $2.02+0$ \\
\hline$(18$ & $7.99-3$ & $4.23+0$ & $4.02+0$ & $3.80+0$ & $3.56+0$ & $3.29+0$ & $2.96+0$ & $2.60+0$ & $2.25+0$ & $1.98+0$ & $1.68+0$ \\
\hline $4,3,1)$ & $1.24-6$ & $1.88+0$ & $1.88+0$ & $1.95+0$ & $2.00+0$ & $1.97+0$ & $1.86+0$ & $1.69+0$ & $1.52+0$ & $1.41+0$ & $1.29+0$ \\
\hline$(18,14,3,2)$ & $2.72-2$ & $9.52+0$ & $9.27+0$ & $9.13+0$ & $8.94+0$ & $8.55+0$ & $7.88+0$ & $7.04+0$ & $6.24+0$ & $5.66+0$ & +0 \\
\hline$(18,14,4,1)$ & $3.50-5$ & $4.79-1$ & $4.10-1$ & $3.80-1$ & $3.64-1$ & $3.52-1$ & $3.49-1$ & $3.64-1$ & $3.99-1$ & $4.47-1$ & $4.95-1$ \\
\hline$(18,14,4,2)$ & $2.04-1$ & $1.44+0$ & $1.23+0$ & $1.14+0$ & $1.09+0$ & $1.06+0$ & $1.05+0$ & $1.09+0$ & $1.20+0$ & $1.34+0$ & $1.48+0$ \\
\hline $4,4,3)$ & $4.76-1$ & $2.39+0$ & $2.05+0$ & $1.90+0$ & $1.82+0$ & $1.76+0$ & $1.74+0$ & $1.82+0$ & $2.00+0$ & $2.24+0$ & $2.47+0$ \\
\hline & $0.00+0$ & $4.47-2$ & $5.16-2$ & $5.71-2$ & $5.98-2$ & $6.09-2$ & $6.19-2$ & $6.48-2$ & $7.06-2$ & $7.74-2$ & $7.91-2$ \\
\hline$, 5,2)$ & $6.55+0$ & $1.34-1$ & $1.55-1$ & $1.71-1$ & 1.7 & $1.83-1$ & $1.86-1$ & $1.94-1$ & $2.12-1$ & $2.32-1$ & $2.37-1$ \\
\hline$(18,14,5,3)$ & $5.69-2$ & $2.23-1$ & $2.58-1$ & $2.85-1$ & $2.99-1$ & $3.05-1$ & $3.10-1$ & $3.24-1$ & $3.53-1$ & $3.87-1$ & $3.95-1$ \\
\hline$(18,14,5,4)$ & $3.29+0$ & $2.05+0$ & $1.86+0$ & $1.74+0$ & $1.68+0$ & $1.64+0$ & $1.62+0$ & $1.63+0$ & $1.68+0$ & $1.74+0$ & $1.74+0$ \\
\hline$(20,14,2,1)$ & $7.66-2$ & $1.27+0$ & $1.27+0$ & $1.28+0$ & $1.26+0$ & $1.20+0$ & $1.15+0$ & $1.22+0$ & $1.37+0$ & $1.51+0$ & $1.59+0$ \\
\hline$(20,14,3,1)$ & $1.78-5$ & $9.66-1$ & $1.00+0$ & $1.03+0$ & $1.02+0$ & $9.88-1$ & $9.96-1$ & $2+0$ & +0 & $5+0$ & $5+0$ \\
\hline & $1.95-1$ & $3.76+0$ & $3.84+0$ & $3.93+0$ & $3.88+0$ & $3.72+0$ & $3.68+0$ & $4.04+0$ & $4.66+0$ & $5.15+0$ & $5.25+0$ \\
\hline$(20,14,4,1)$ & $1.44-4$ & $6.29-1$ & $5.27-1$ & $4.50-1$ & $4.18-1$ & $4.32-1$ & $4.67-1$ & $4.89-1$ & $4.87-1$ & $4.65-1$ & $3.90-1$ \\
\hline$(20,14,4,2)$ & $1.19+0$ & $1.89+0$ & $1.58+0$ & $1.35+0$ & $1.25+0$ & $1.29+0$ & $1.40+0$ & $1.47+0$ & $1.46+0$ & $1.39+0$ & $1.17+0$ \\
\hline$(20,14,4,3)$ & $2.41+0$ & $3.15+0$ & $2.63+0$ & $2.25+0$ & $2.09+0$ & $2.16+0$ & $2.33+0$ & $2.45+0$ & $2.43+0$ & $2.32+0$ & $1.95+0$ \\
\hline$(20,14,5,1)$ & $0.00+0$ & $6.78-2$ & $8.33-2$ & $1.00-1$ & $1.09-1$ & $1.06-1$ & $9.60-2$ & $8.46-2$ & $7.55-2$ & $6.87-2$ & $5.59-2$ \\
\hline$(20$ & $3.32+1$ & $2.04-1$ & $2.50-1$ & $3.00-1$ & $3.27-1$ & $3.19-1$ & $2.88-1$ & $2.54-1$ & $2.27-1$ & $2.06-1$ & $1.68-1$ \\
\hline & $2.19-1$ & $3.39-1$ & $4.17-1$ & $5.01-1$ & $6-1$ & $5.32-1$ & $4.80-1$ & $4.23-1$ & $3.78-1$ & $3.43-1$ & $2.79-1$ \\
\hline$(20,14,5,4)$ & $4.49+0$ & $5.96-1$ & $6.37-1$ & $6.45-1$ & $6.30-1$ & $6.13-1$ & $6.29-1$ & $7.20-1$ & $8.82-1$ & $1.05+0$ & $1.20+0$ \\
\hline$(18,16,2,1)$ & $3.08-2$ & $3.71+0$ & $3.50+0$ & $3.34+0$ & $3.22+0$ & $3.14+0$ & $3.09+0$ & $3.12+0$ & $3.21+0$ & $3.32+0$ & $3.16+0$ \\
\hline$(18,16,3,1)$ & $2.37-6$ & $7.23-1$ & $6.95-1$ & $6.76-1$ & $6.65-1$ & $6.60-1$ & $6.71-1$ & $7.15-1$ & $7.81-1$ & $8.54-1$ & $8.98-1$ \\
\hline & $5.17-3$ & $1.67+0$ & $1.55+0$ & $1.45+0$ & $8+0$ & $1.32+0$ & +0 & +0 & +0 & +0 & +0 \\
\hline 1) & $3.14-1$ & $2.61+0$ & $2.67+0$ & $2.71+0$ & $2.72+0$ & $2.70+0$ & $2.66+0$ & $2.62+0$ & $2.60+0$ & $2.58+0$ & $2.30+0$ \\
\hline$(18,16,4,2)$ & $8.23-2$ & $1.57+0$ & $1.60+0$ & $1.63+0$ & $1.63+0$ & $1.62+0$ & $1.60+0$ & $1.57+0$ & $1.56+0$ & $1.55+0$ & $1.39+0$ \\
\hline$(18,16,4,3)$ & $2.21-5$ & $5.23-1$ & $5.35-1$ & $5.42-1$ & $5.44-1$ & $5.39-1$ & $5.32-1$ & $5.24-1$ & $5.19-1$ & $5.18-1$ & $4.65-1$ \\
\hline$(18,16,5,1)$ & $4.17-2$ & $4.73-1$ & $4.93-1$ & $4.93-1$ & $4.82-1$ & $4.71-1$ & $4.63-1$ & $4.58-1$ & $4.52-1$ & $4.44-1$ & $3.84-1$ \\
\hline & $3.91+0$ & $2.84-1$ & $2.96-1$ & $2.96-1$ & $2.89-1$ & $2.83-1$ & $2.78-1$ & $2.75-1$ & $2.71-1$ & $2.64-1$ & $2.21-1$ \\
\hline & $0.00+0$ & $9.46-2$ & $9.87-2$ & $9.86-2$ & $9.65-2$ & $9.42-2$ & $9.27-2$ & $9.15-2$ & $9.01-2$ & $8.76-2$ & $7.20-2$ \\
\hline$(18,16,5,4)$ & $2.59+0$ & $1.58+0$ & $1.48+0$ & $1.37+0$ & $1.29+0$ & $1.25+0$ & $1.23+0$ & $1.21+0$ & $1.18+0$ & $1.18+0$ & $1.25+0$ \\
\hline$(20,16,2,1)$ & $3.10-1$ & $2.65+0$ & $2.37+0$ & $2.22+0$ & $2.18+0$ & $2.22+0$ & $2.30+0$ & $2.44+0$ & $2.68+0$ & $3.00+0$ & $3.35+0$ \\
\hline$(20,16,3,1)$ & $3.67-5$ & $6.44-1$ & $5.86-1$ & $5.69-1$ & $5.86-1$ & $6.13-1$ & $6.48-1$ & $7.04-1$ & $7.89-1$ & $8.87-1$ & $9.73-1$ \\
\hline$(20,16,3,2)$ & $3.54-2$ & $9.64-1$ & $8.42-1$ & $7.48-1$ & $6.92-1$ & $6.72-1$ & $6.75-1$ & $6.88-1$ & $7.28-1$ & $8.06-1$ & $9.30-1$ \\
\hline$(20,16,4,1)$ & $1.90+0$ & $1.77+0$ & $1.61+0$ & $1.52+0$ & $1.53+0$ & $1.60+0$ & $1.71+0$ & $1.86+0$ & $2.05+0$ & $2.22+0$ & $2.13+0$ \\
\hline & $4.26-1$ & $1.06+0$ & $9.67-1$ & $9.12-1$ & $9.20-1$ & $9.61-1$ & $1.03+0$ & $1.12+0$ & $1.23+0$ & $1.33+0$ & $1.27+0$ \\
\hline$(20,16,4,3)$ & $8.42-5$ & $3.53-1$ & $3.22-1$ & $3.04-1$ & $3.06-1$ & $3.20-1$ & $3.42-1$ & $3.73-1$ & $4.11-1$ & $4.43-1$ & $4.25-1$ \\
\hline$(20,16,5,1)$ & $1.45-1$ & $8.13-2$ & $9.11-2$ & $1.10-1$ & $1.50-1$ & $2.11-1$ & $2.90-1$ & $3.83-1$ & $4.58-1$ & $4.83-1$ & $3.96-1$ \\
\hline$(20,16,5,2)$ & $2.31+1$ & $4.88-2$ & $5.47-2$ & $6.61-2$ & $8.99-2$ & $1.26-1$ & $1.74-1$ & $2.30-1$ & $2.75-1$ & $2.90-1$ & $2.37-1$ \\
\hline$(20,16,5,3)$ & $0.00+0$ & $1.63-2$ & $1.82-2$ & $2.20-2$ & $3.00-2$ & $4.21-2$ & $5.80-2$ & $7.67-2$ & $9.16-2$ & $9.66-2$ & $7.91-2$ \\
\hline$(20,16,5,4)$ & $3.73+0$ & $1.10+0$ & $1.21+0$ & $1.29+0$ & $1.34+0$ & $1.36+0$ & $1.35+0$ & $1.36+0$ & $1.38+0$ & $1.40+0$ & $1.27+0$ \\
\hline
\end{tabular}

